\title{
Multiplexing holograms in an acrylamide photopolymer
}

\author{
Elena Fernández*a ${ }^{* a}$ Manuel Ortuño ${ }^{\mathrm{b}}$, Andrés Márquez $^{\mathrm{b}}$, Sergi Gallego ${ }^{\mathrm{b}}$, and Inmaculada Pascual ${ }^{\mathrm{a}}$ \\ ${ }^{a}$ Dept. Interuniversitario de Óptica, Universidad de Alicante, Apartado 99, E-03080 Alicante, Spain \\ ${ }^{\mathrm{b}}$ Dept. de Física, Ing. de Sistemas y Ta de la Señal, Universidad de Alicante, Ap. 99, E-03080 \\ Alicante, Spain \\ *elena.fernandez@ua.es
}

\begin{abstract}
A peristrophic multiplexing method is used to store various diffraction gratings at the same spot in the material. This material is formed of acrylamide photopolymers which are considered interesting materials for recording holographic memories. They have high diffraction efficiency (ratio between diffracted and incident beams), high energetic sensitivity and optical quality, and developing processes are not necessary. In this work, the photopolymer is composed of acrylamide (AA) as the polymerizable monomer, triethanolamine (TEA) as radical generator, N,N'methylene-bisacrylamide (BMA) as crosslinker, yellowish eosin (YE) as sensitizer and a binder of polyvinyl alcohol (PVA) ${ }^{1}$. The layers of material obtained are approximately $1 \mathrm{~mm}$ thick. Using holographic recording schedules, the exposure energy each hologram should receive in order to achieve uniform diffraction efficiency is optimized. The purpose of these recording schedules is to enable full advantage to be taken of the whole dynamic range of the material and to share it between the individual holograms. The Scheduled Exposure Method (SEM) and the Incremental Exposure Method (IEM) are the two multiplexing schedules used to determine the recording times ${ }^{2}$. Having determined these times, the results obtained with both methods are compared to ascertain which method enables the greatest number of holograms to be recorded with the highest, most uniform diffraction efficiencies.
\end{abstract}

Keywords: Photopolymers, Peristrophic multiplexing, Holographic recording materials.

\section{INTRODUCTION}

We live in an age of information science and technology in which the use of computers for information processing and storage has become essential. After CD-ROM's, with a capacity to store $0.7 \mathrm{bits} / \mu^{2}$, and DVD's, with a capacity to store $4.5 \mathrm{bits} / \mu_{\mathrm{m}}^{2}$, a new field of research has been opened up in three-dimensional holographic discs $\mathrm{s}^{3-7}$. Investigations are centred on the characterisation of new holographic recording materials ${ }^{2}$ and the development of new multiplexing schedules in order to store information with holographic techniques. The aim of new techniques is to enable the maximum number of bits $/ \mu \mathrm{m}^{2}$ to be stored. InPhase ${ }^{8}$ has managed to store $150 \mathrm{bits} / \mu \mathrm{m}^{2}$. For this reason, different methods, such as angular ${ }^{1,6,8-9}$, peristrophic ${ }^{10-11}$, or shift-multiplexing ${ }^{12-13}$ are being made use of to store multiple holograms at the same location.

In this respect, photopolymers are considered interesting materials for recording holographic memories because they have excellent holographic characteristics, such as high refractive index, good light sensitivity, real time image development, high optical quality, low cost and high dynamic range ${ }^{2,6,14}$ (this parameter describes the capacity of the material to store many holograms at the same location with a higher diffraction efficiency). In addition, their properties like energetic sensitivity or spectral sensitivity can be easily changed by modifying the composition ${ }^{1-2,15}$.

The material used to carry out this experiment is a photopolymer based on PVA-acrylamide ${ }^{1,15}$. Layers about $800 \mu \mathrm{m}$ thick were made, and when 60 holograms are stored, a dynamic range of $\mathrm{M} \#=11$ is obtained in them.

In order to fully exploit the diffraction efficiency of the material used, as many holograms as possible need to be stored.

Photon Management II, edited by John T. Sheridan, Frank Wyrowski, Proc. of SPIE Vol. 6187,

618712, (2006) $\cdot 0277-786 \mathrm{X} / 06 / \$ 15 \cdot$ doi: $10.1117 / 12.662400$

Proc. of SPIE Vol. 6187 618712-1 
In this study, a peristrophic multiplexing method is used in order to increase the number of holograms that may be stored at the same location (the axis is perpendicular to the sample at the intersection of the beams).

Using holographic recording schedules ${ }^{11,13}$, the exposure energy each hologram should receive in order to achieve a uniform diffraction efficiency is optimised. The purpose of these recording schedules is to enable full advantage to be taken of the whole dynamic range of the material and to share it between the individual holograms. The Scheduled Exposure Method ${ }^{11}$ (SEM) and the Incremental Exposure Method ${ }^{13}$ (IEM) are the two multiplexing schedules used to determine the optimum exposure times to obtain more uniform diffraction efficiency. Having determined these times, the results obtained with both methods are compared to ascertain which method enables the greatest number of holograms to be recorded with the highest, most uniform diffraction efficiencies.

\section{EXPERIMENTAL}

\subsection{Preparation of the material}

The holograms are registered in a photopolymer composed of acrylamide (AA) as the polymerizable monomer, triethanolamine (TEA) as radical generator, N,N'methylene-bis-acrylamide (BMA) as crosslinker, yellowish eosin (YE) as sensitizer and a binder of polyvinyl alcohol (PVA) ${ }^{1}$. Introduction of BMA in the composition improves the energetic sensitivity and diffraction efficiency of the material and, in addition, gives a greater stability to the stored grating, thereby preventing it from disappearing with time. Table 1 shows the component concentrations of the photopolymer composition.

Table 1: Concentrations of the photopolymer composition.

\begin{tabular}{cc}
\hline & Composition \\
\hline Polyvinylalcohol & $13.50 \% \mathrm{w} / \mathrm{v}$ \\
Acrylamide & $0.31 \mathrm{M}$ \\
Triethanolamine & $0.12 \mathrm{M}$ \\
Yellowish eosin & $9 \cdot 10^{-5} \mathrm{M}$ \\
N,N'methylene-bis-acrylamide & $0.04 \mathrm{M}$ \\
\hline
\end{tabular}

A solution of PVA in water forms the matrix and this is used to prepare the mixture of AA, BMA, and photopolymerization initiator system composed of TEA and YE. The mixture is made under red light, deposited by gravity in circular polystyrene molds and left in the dark for six days to allow the water to evaporate in conditions of temperature, $\mathrm{T}$, between $20^{\circ} \mathrm{C}$ and $25{ }^{\circ} \mathrm{C}$, and relative humidity, $\mathrm{RH}, 40 \%-60 \%$. These conditions of drying time, temperature and relative humidity are optimized to obtain the maximum diffraction efficiency in $800 \mu \mathrm{m}$ thick plates. Once dry, they are removed from the mold and placed in a glass support to be used for recording.

\subsection{Holographic set-up}

Holographic gratings were recorded using the output from a diode-pumped frequency-doubled ND:YAG laser (Coherent Verdi V2) which was split into two beams and then spatially filtered, using a microscope objective lens and a pinhole, and collimated to yield a plane-wave source of light at $532 \mathrm{~nm}$. The diameters of these beams are $1.5 \mathrm{~cm}$ with an intensity ratio of 1:1. The beam intensity that is used depends on the exposure schedule method. In SEM the sum of the total intensity of the two recording beams is $5 \mathrm{~mW} / \mathrm{cm}^{2}$, and in IEM, it is $0.6 \mathrm{~mW} / \mathrm{cm}^{2}$. The Gaussian light beams were spatially overlapped at the recording medium intersection at an angle of $17.4^{\circ}$ (measured in air), resulting in an interference grating with a spatial frequency of 1125 lines $/ \mathrm{mm}$. The diffracted intensity is monitored in real time with the He-Ne laser positioned at Bragg's angle $\left(\theta^{\prime}=20.8^{\circ}\right)$. In order to obtain diffraction efficiency as a function of the angle at reconstruction, the plates are placed on a rotating stage. The diffraction efficiency was calculated as the ratio of the diffracted beam to the incident power (Fig. 1). 


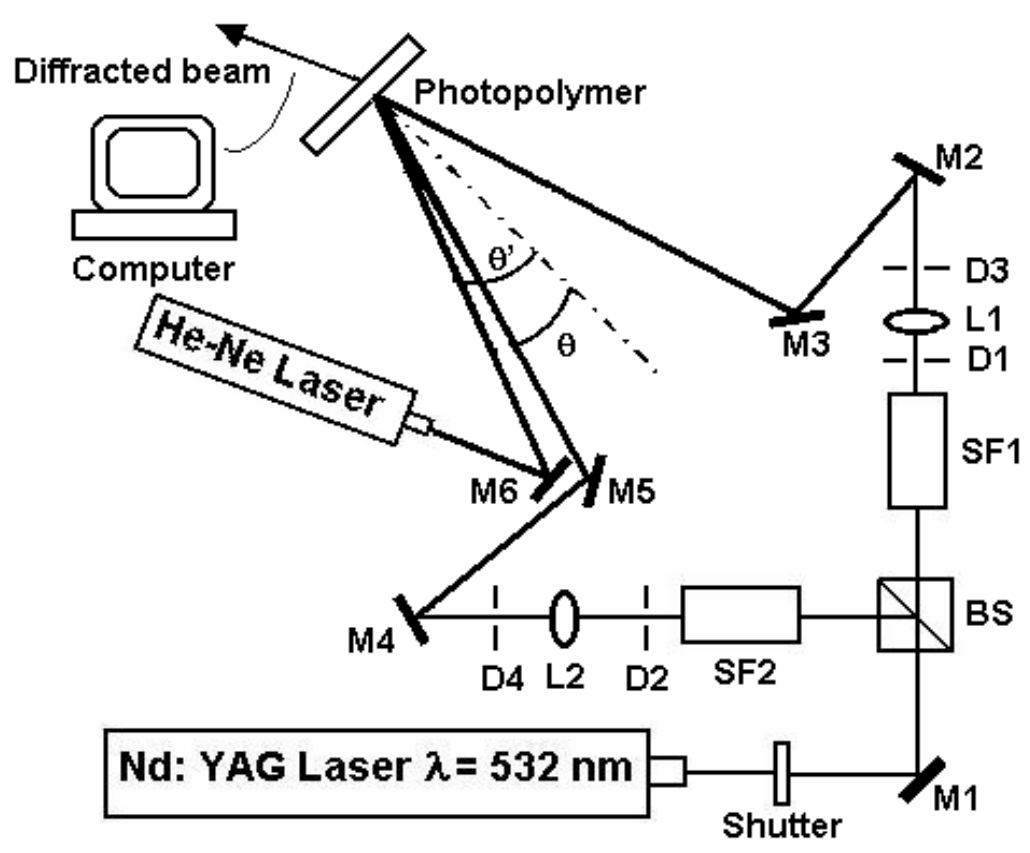

Figure 1: Experimental setup: Mi mirror, Li lens, Di, diaphragm, SFi, microscope objective lens and pinhole.

\section{RESULTS}

\subsection{Schedule Exposure Method (SEM)}

In the first experiment, 60 holograms are stored at the same location using the SEM. The peristrophic multiplexing method is used to store the holograms. Holograms are stored with a separation of 3 degrees. This angular separation for peristrophic multiplexing is chosen because we do not want the holograms to overlap. The sum of the total intensity of the two recording beams is $5 \mathrm{~mW} / \mathrm{cm}^{2}$, with an intensity ratio of $1: 1$.

In order to store the 60 holograms, the exposure time to be used to record them must be decided upon. As the holograms are stored, the monomer and dye in the material are consumed. This means that a greater exposure energy is necessary if the last holograms recorded are to have the same diffraction efficiency as the first. Therefore, the exposure time used to store the holograms is as follows: 1 second for the first 10 holograms, and then half a second is added for every 5 holograms stored.

Fig. 2 shows the diffraction efficiency obtained when the holograms are recorded with the above exposure times versus the number of holograms stored. Sixty holograms were stored with a mean diffraction efficiency of $3.3 \%$. As can be seen, the diffraction efficiency of the first five holograms is much higher than the mean. This is due to the fact that at the beginning, the material is very sensitive to the exposure energy used. As the holograms are recorded, the monomer and dye in the material are consumed and this means that greater exposure energy is necessary to reach the same diffraction efficiency, as mentioned above. Therefore, the last holograms stored have a lower diffraction efficiency since, as all the monomer and dye have been consumed, the hologram will not be formed even though the exposure energy is increased. 


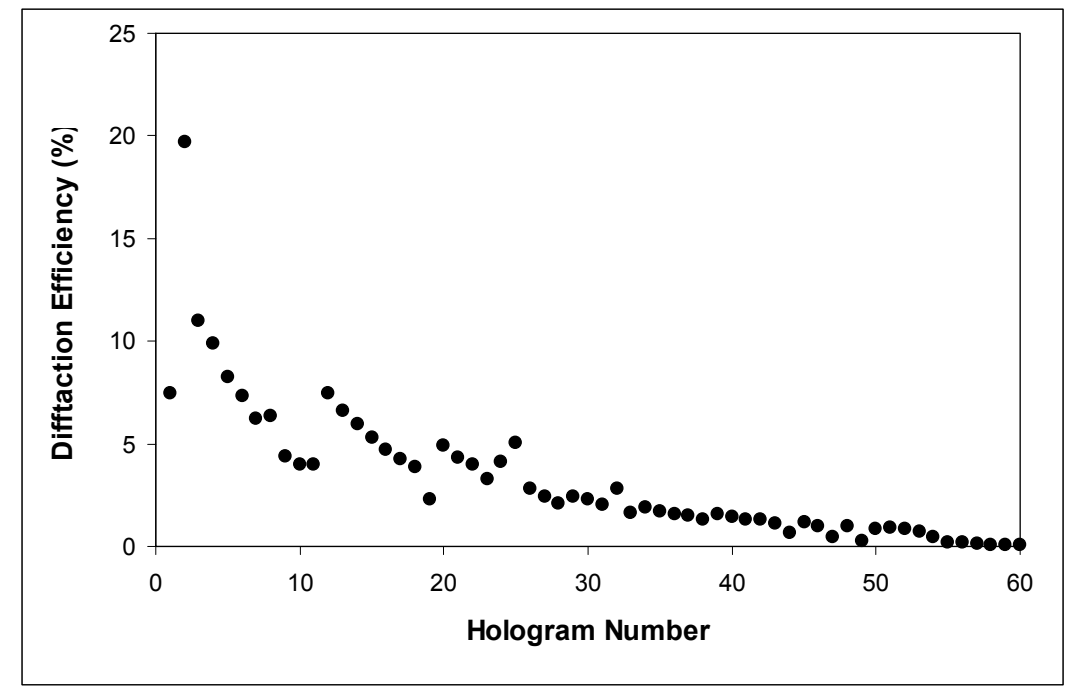

Figure2: Diffraction efficiency versus hologram number before SEM.

From the diffraction efficiencies reached in the stored gratings we calculated the cumulative grating strength, $\sum_{i=1}^{N} \eta_{i}^{1 / 2}$, where $\eta$ is the diffraction efficiency and $\mathrm{N}$ the number of holograms stored so far, and represented it as a function of exposure energy (Fig. 3). When the curve is saturated, the dynamic range is M\# $=9.5$. From the dynamic range and the number of holograms to be stored, it is possible to determine the mean theoretical diffraction efficiency that could be reached for each of the holograms, Eq. (1). Therefore, when $M \#=9.5$ and 60 holograms are stored, the mean theoretical diffraction efficiency is $2.5 \%$. In other words, if 60 holograms were stored with the same diffraction efficiency, their mean diffraction efficiency would be $2.5 \%$. If the experimental diffraction efficiency is higher than the theoretical diffraction efficiency it is because the first 20 holograms were stored with a very high diffraction efficiency.

$$
\eta_{\text {medio }}=\left(\frac{\mathrm{M \#}}{\mathrm{N}}\right)^{2}
$$

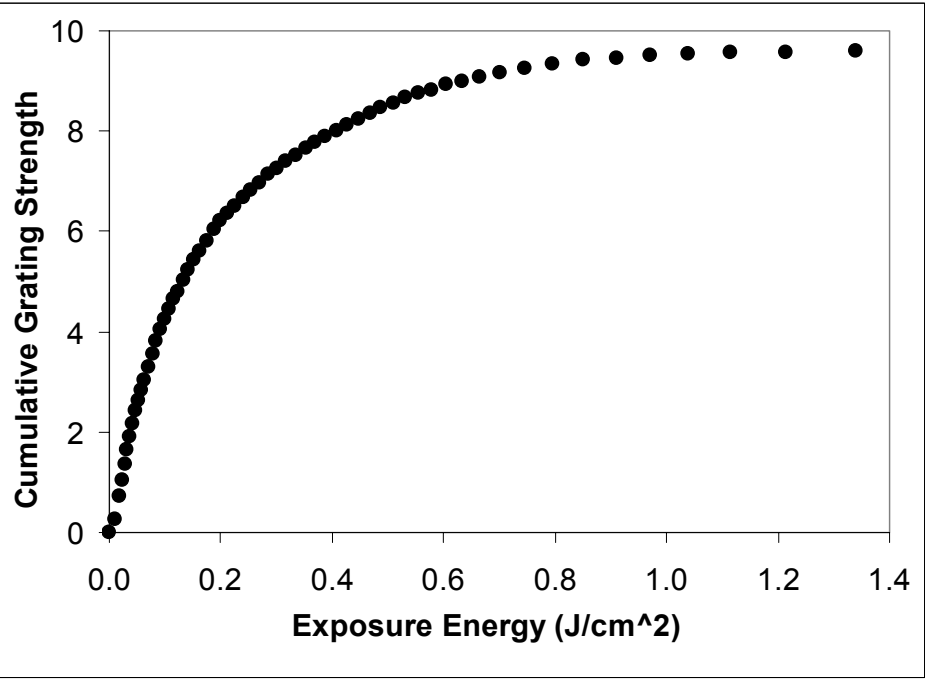

Figure 3: Cumulative grating strength as a function of exposure energy. 
Then, in order to store the 60 holograms with the same diffraction efficiency, the SEM is used to optimize the exposure energy that each hologram should receive in order to achieve the same diffraction efficiency ${ }^{11}$. This is done as follows: first, the data obtained from Fig. 3 are fitted in the following theoretical equation

$$
A=a_{0}+a_{1} E+a_{2} E^{2}+a_{3} E^{3}+a_{4} E^{4}+a_{5} E^{5}+a_{6} E^{6}
$$

where $\mathrm{A}$ is the cumulative grating strength and $\mathrm{E}$ the exposure energy. Once the coefficients $\mathrm{a}_{\mathrm{i}}$ have been calculated, the time needed to record the holograms may be calculated from the equation.

$$
\begin{aligned}
t_{n}= & A_{\text {Sat }} / N \cdot I\left[a_{1}+2 a_{2} \sum_{i=1}^{n-1} E_{i}+3 a_{3}\left(\sum_{i=1}^{n-1} E_{i}\right)^{2}\right. \\
& \left.+4 a_{4}\left(\sum_{i=1}^{n-1} E_{i}\right)^{3}+5 a_{5}\left(\sum_{i=1}^{n-1} E_{i}\right)^{4}+6 a_{6}\left(\sum_{i=1}^{n-1} E_{i}\right)^{5}\right]
\end{aligned}
$$

where $A_{\text {sat }}$ is the dynamic range obtained, $N$ is the number of holograms to be stored, $\mathrm{I}$ is the recording intensity, and $\mathrm{E}_{\mathrm{i}}$ the energy used to record up to the i'th hologram.

Fig. 4 shows the diffraction efficiency of the 60 stored holograms versus the hologram number after the SEM is used. In this case, the dynamic range is $\mathrm{M} \#=11$, and the mean diffraction efficiency is $3.5 \%$. The value of M\# has increased because the dynamic range has been more fully exploited thanks to the new recording times. The results in Fig. 4 are better than in Fig. 2 because the diffraction efficiency of all the holograms is more uniform and nearer the mean diffraction efficiency. The results in Fig 4 have been represented with the same scale that Fig. 2 to compare them better.

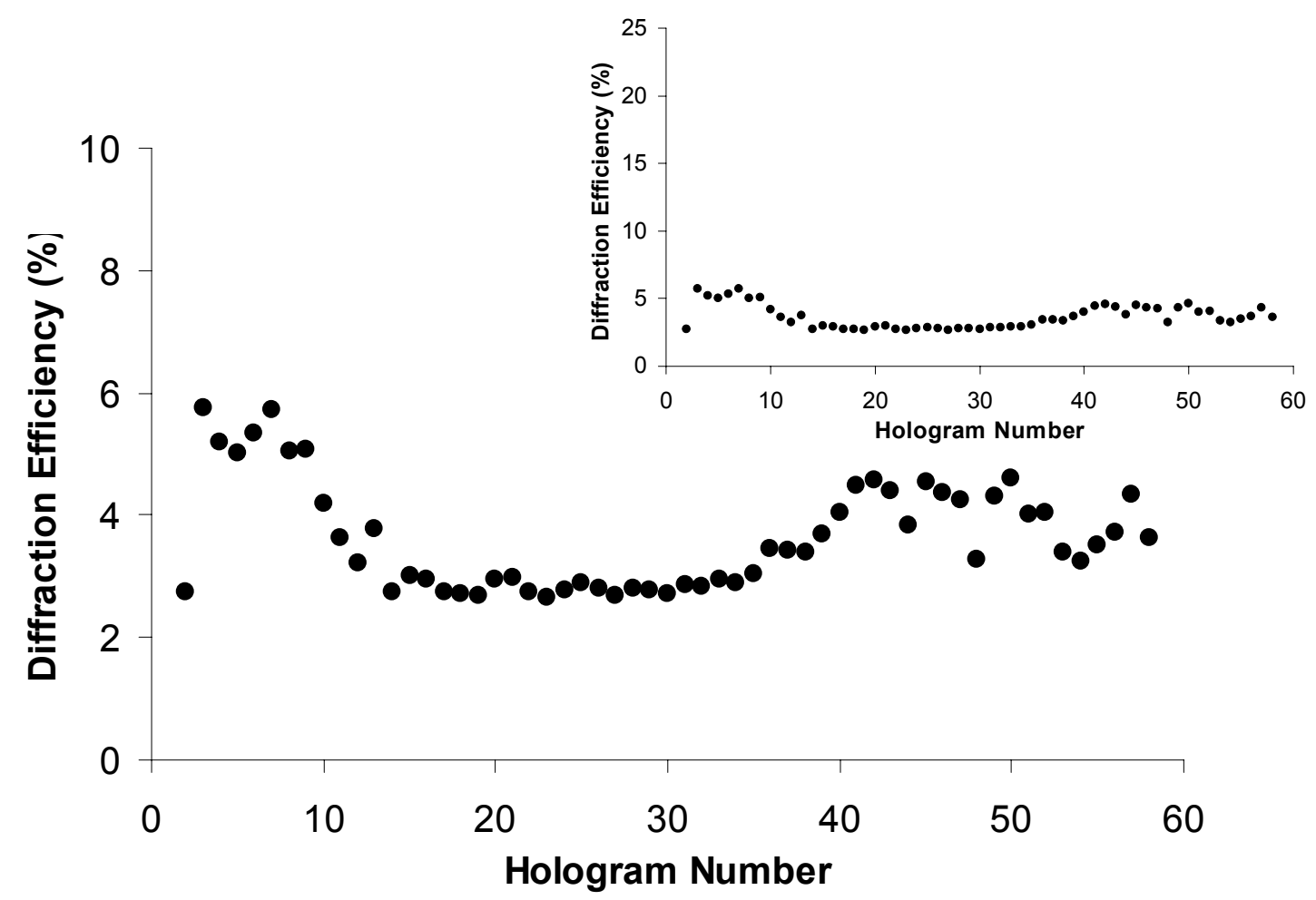

Figure 4: Diffraction efficiency versus hologram number after SEM with two different scales. 


\subsection{The Incremental Exposure Method (IEM)}

In the second experiment, 60 holograms are stored using the IEM. We use the peristrophic multiplexing method and the holograms are separated by 3 degrees, just as in the previous method. The sum of the total intensity of the two recording beams is $0.6 \mathrm{~mW} / \mathrm{cm}^{2}$, with an intensity ratio of $1: 1$.

As mentioned above, if all the holograms are stored with the same exposure energy, the diffraction efficiency of the first holograms stored is higher than that of the last ones. This is because the monomer and the dye are consumed as the holograms are stored.

This second method consists in storing the holograms with the minimum exposure energy necessary to form the holograms, but it must be low enough so that no holograms are registered with a diffraction efficiency higher than that of the others.

The results of storing 60 holograms with this method are shown in Fig. 5. As can be seen, the diffraction efficiency of the 60 holograms is quite uniform. All the values oscillate around the mean diffraction efficiency, that is $0.25 \%$, but the dynamic range does not surpass $\mathrm{M} \#=2.5$. The material is the same as that used in the previous experiment $(\mathrm{M} \#=11)$. If a greater M\# has not been obtained, it is because all the available dynamic range has not been exploited. Therefore, with this method it is possible to achieve much more uniform values of diffraction efficiency, although the values obtained are not very high. However, it would be possible to store many more holograms if all the dynamic range were used.

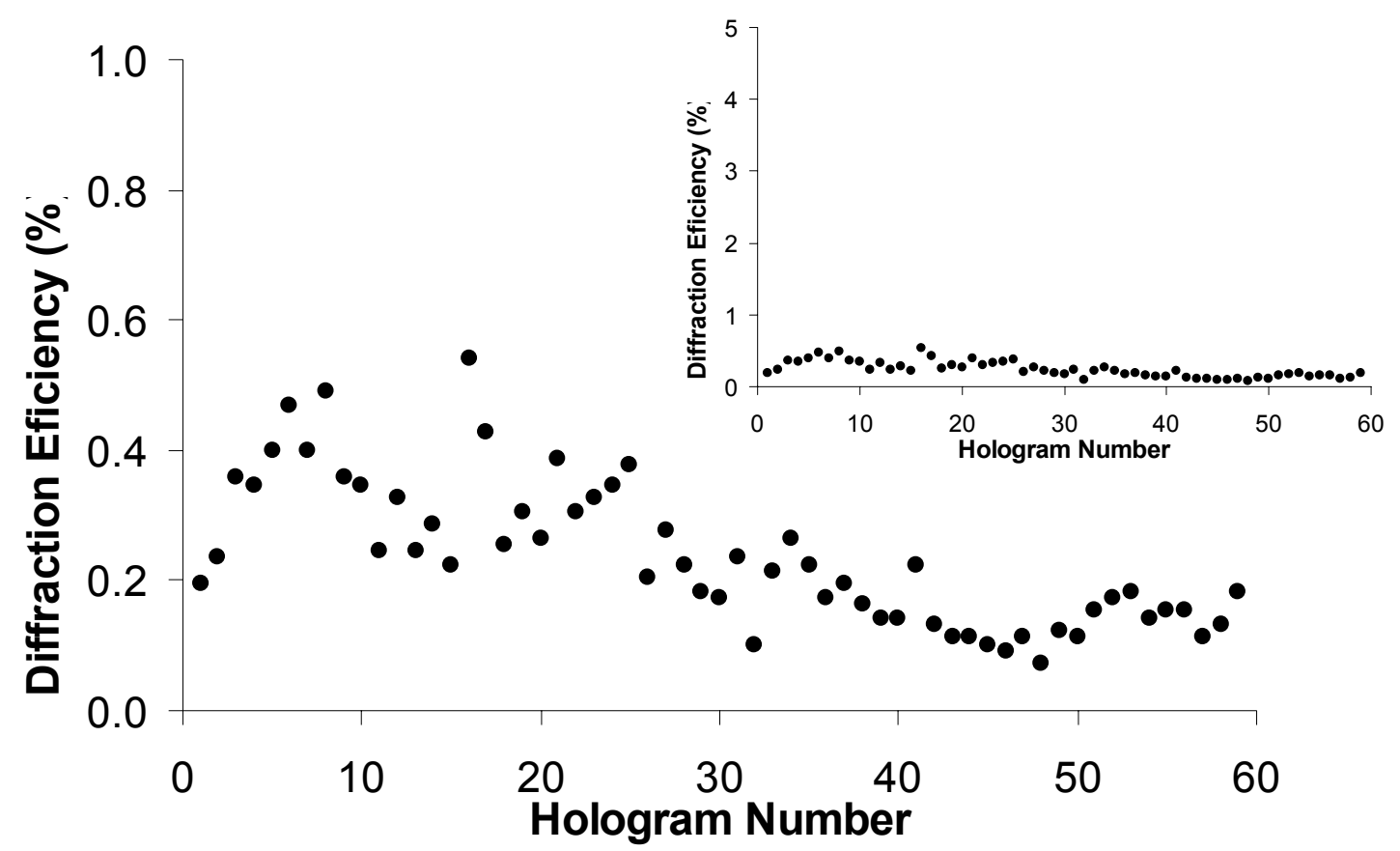

Figure 5: Diffraction efficiency versus hologram number after IEM with two different scales. 


\section{CONCLUSION}

Two methods have been used to achieve more uniform values of the diffraction efficiency of 60 stored holograms. With the first method, SEM, values of diffraction efficiency of between 3 and $4.5 \%$ are obtained with an $\mathrm{M} \#=11$. These values are not more uniform but they are high. With the second method, IEM, diffraction efficiencies between 0.1 and $0.4 \%$ are obtained. These values are more uniform but they are not very high. In addition, not all the dynamic range is used $(\mathrm{M} \#=2.5)$. Thus, according to Eq. 1, more than 150 holograms could be stored with the IEM.

\section{ACKNOWLEDGMENT}

This work was supported by the "Ministerio de Educación y Ciencia"(Spain) under projects FIS2005-05881-C02-01 and FIS2005-05881-C02-02.

\section{REFERENCES}

1. M. Ortuño, S. Gallego, C. García, C. Neipp, A. Beléndez, I. Pascual, "Optimization of a $1 \mathrm{~mm}$ thick PVA/acrylamide recording material to obtain holographic memories: method of preparation and holographic properties”, Appl. Phys. B 76, 851, (2003).

2. Ken Y. Hsu, Shiuan Huei Lin, Yi-Nan Hsiao, "Experimental characterization of phenanthrenequinode-doped poly(methyl methacrylate) photopolymer for volume holographic storage", Opt. Eng. 42, 1390-1396, (2003).

3. A. Pu, D. Psaltis, "High-density recording in photopolymer-based holographic three-dimensional disks", Appl. Opt. 35, 2389-2398, (1996).

4. O. Graydon, editor, "Holographic storage turns blue", Opto \& Laser Europe 125, 7, (2005).

5. A. H. Tullo, "Data storage in 3-D", Chemical \& Engineering News 83, number 26, 31-32, (2005).

6. M. Schnoes, B. Ihas, A. Hill, L. Dhar, D. Michaels, S. Setthachayanon, G. Schomberger, W. L. Wilson, "Holographic Storage Media for Practical Systems" in Practical Holography XVII and Holographic Materials IX, Tung H. Jeong, ed., Proc SPIE 5005, 29-37, (2003).

7. R. R. McLeod, A. J. Daiber, M. E. McDonald, T. L. Robertson, T. Slagle, S. L. Sochava, L. Hesselink, "Microholographic optical disk data storage", Appl. Opt. 44, 3197-3207, (2005).

8. H. Sherif, I. Naydenova, S. Martin, C. McGinn, "Characterization of an acrylamide-based photopolymer for data storage utilizing holographic angular multiplexing", Journal of Optics A: Pure and Appl. Opt. 7, 255-260, (2005).

9. I.El Hafidi, R. Grzymala, R. Kiefer, L. Elouad, P. Meyrueis, "Optical data storage on protein using angular multiplexing", Optics \& Laser Technology 37, 503-508, (2005).

10. K. Curtis, A. Pu, D. Psaltis, "Method for holographic storage using peristrophic multiplexing", Opt. Lett. 19, 993994, (1994).

11. A. Pu, K. Curtis, D. Psaltis, "Exposure schedule for multiplexing holograms in photopolymer films", Opt. Eng. 35, 2824-2828, (1996).

12. G.A. Rakuljic, V. Levya, A. Yariv, "Optical data storage by using orthogonal wavelength-multiplexed volume holograms”, Opt. Lett. 17, 1471-1473, (1992).

13. Liangcai Cao, Xiaosu Ma, Qingsheng He, Hua Wu, Guofan Jin, "Imaging spectral device based on multiple volume holographic gratings", Opt. Eng. 43, 2009-2016, (2004).

14. Fai H. Mok, G. W. Burr and D. Psaltis, "System metric for holographic memory systems", Opt. Lett. 21, 896-898, (1996).

15. S. Gallego, M. Ortuño, C. Garcia, C. Neipp, A. Belendez, I. Pascual, "High-efficiency volume holograms recording on acrylamide and N,N'methilene-bis-acrylamide photopolymer with pulsed láser", J. Mod. Opt. 52, 1575-1584, (2005). 